TITLE:

\title{
Multimodal evaluation of macular function in age-related macular degeneration.
}

\section{$\operatorname{AUTHOR}(S)$ :}

Ogino, Ken; Tsujikawa, Akitaka; Yamashiro, Kenji; Ooto, Sotaro; Oishi, Akio; Nakata, Isao; Miyake, Masahiro; Takahashi, Ayako; Ellabban, Abdallah A; Yoshimura, Nagahisa

\section{CITATION:}

Ogino, Ken ...[et al]. Multimodal evaluation of macular function in age-related macular degeneration.. Japanese journal of ophthalmology 2014, 58(2): 155-165

\section{ISSUE DATE:}

2014-03

URL:

http://hdl.handle.net/2433/199875

\section{RIGHT:}

The final publication is available at Springer via http://dx.doi.org/10.1007/s10384-0130295-Z:; この論文は出版社版でありません。引用の際には出版社版をご確認ご利用くだ さい。; This is not the published version. Please cite only the published version. 
3 Ken Ogino, Akitaka Tsujikawa, Kenji Yamashiro, Sotaro Ooto, Akio Oishi, Isao Nakata, Masahiro Miyake, Ayako

8 Running head: Macular function in AMD

10 Corresponding author: Akitaka Tsujikawa, Department of Ophthalmology and Visual Sciences, Kyoto University

11 Graduate School of Medicine, Sakyo-ku, Kyoto 606-8507, Japan; tel: +81-75-751-3250, fax: +81-75-752-0933;

12 e-mail: tujikawa@kuhp.kyoto-u.ac.jp

13 Word counts 249 in the abstract and 2712 in the text.

14 This manuscript includes 38 references, 7 figures, and 2 tables

15 The authors have no conflicts of interest in the materials or devices mentioned in the article. 


\section{ABSTRACT}

17 Objective To evaluate macular function using multimodality in eyes with age-related macular degeneration (AMD) at

18 various stages.

19 Methods Macular function in 20 control eyes (20 subjects), 17 eyes (17 patients) with large drusen, 18 eyes (18

20 patients) with drusenoid pigment epithelial detachment (PED), and 19 eyes (19 patients) with neovascular AMD was

21 examined using a Landolt chart for visual acuity; retinal sensitivity was measured by microperimetry; and focal

22 macular electroretinography (fmERG) was performed. In all of these eyes, retinal morphology was examined using

23 optical coherence tomography.

24 Results Eyes with neovascular AMD showed morphologic changes in the neurosensory retina as well as marked

25 deterioration of macular function in all parameters measured with a Landolt chart, fmERG, and microperimetry. Eyes

26 with large drusen showed only minimal morphologic changes in the neurosensory retina. In this large drusen group,

27 although retinal sensitivity at the central point was significantly decreased $(P=0.0063)$, the other parameters of

28 macular function were well preserved. In eyes with drusenoid PED, the structure of the neurosensory retina was well

29 preserved, while the foveal thickness was significantly increased $(P=0.013)$. The macular function of these eyes

30 was significantly deteriorated, with the VA, amplitude of the a-wave and b-wave, and retinal sensitivity being

31 markedly decreased. In addition, the area of PED correlated with the latency of the a- wave and b-wave and with the

32 retinal sensitivity within the central $4^{\circ}$ or $8^{\circ}$ region.

33 Conclusion Multimodal evaluation demonstrated a significant decrease in macular function in drusenoid PED and in 
34 neovascular AMD.

35 Keywords: Age-related macular degeneration, Drusenoid pigment epithelial detachment, Drusen, Focal macular

36 electroretinography, Microperimetry 


\section{Introduction}

39 Age-related macular degeneration (AMD) is one of the leading causes of visual impairment and an intensive

40 therapeutic target in developed countries [1-6]. Drusen or drusenoid pigment epithelium detachment (PED), which is

41 a prodrome lesion of advanced AMD, does not usually cause a severe loss of visual acuity (VA), but it is the

42 subsequent development of choroid neovascularization (CNV) that so often causes the central visual disturbance. So

43 far, however, visual impairment due to AMD has been evaluated primarily by VA measurement alone. Indeed, VA

44 measurement is essential to evaluate visual function, but it reflects only foveal function. Lesions of AMD, including

45 drusen, CNV, serous retinal detachment, subretinal hemorrhage, and PED, are seen not only beneath the fovea but in

46 the larger macular area, which leads to the macular dysfunction.[7]

To evaluate visual function of the entire macular area, simultaneous use of the focal macular electroretinogram

48 (fmERG) and of microperimetry have recently been reported [8, 9]. The fmERG enables measurement of macular

49 function throughout its entirety, even in patients with poor fixation, by monitoring through an infrared camera and

50 manual adjustment of the stimulus to the macular area [10]. Microperimetry allows functional evaluation of selected

51 points throughout the macular area [11, 12]. During this test, the autotracking function corrects for shifts in the

52 measurement position caused by small, involuntary movements. Recent studies using microperimetry have shown

53 that early or advanced AMD often accompanies the severe reduction in sensitivity of the macular area [13-21]. With

54 the use of microperimetry, Yodoi et al reported a functional reduction in the macular area of eyes with subfoveal

55 polypoidal choroidal vasculopathy (PCV), which is a variant of neovascular AMD [22]. In their report, macular 
56 function improved after photodynamic therapy with concomitant recovery of the subjective symptoms, despite there

57 being no improvement in VA.

59 have reported that it is impaired—even in eyes with drusen alone [23, 24]. Indeed, each modality has both

60 advantages and limitations. To evaluate visual function effectively, it would be of help to measure retinal function

61 within the macular area using the multimodality approach. So far, however, little information is available on the

62 multimodal evaluation of visual function in eyes with AMD. Therefore, this study was designed to evaluate the

63 macular function using multimodality in eyes with AMD at various stages, including those with large drusen,

64 drusenoid PED, and those with neovascular AMD.

\section{Patients and methods}

In this prospective study, we performed multimodal evaluation of macular function in eyes with AMD at various

70 Eyes with large drusen were judged by the presence of multiple large drusen (>125 $\mu \mathrm{m})$ within $3000 \mu \mathrm{m}$ of the center of the macula on fundus photographs. The diagnostic criteria of drusenoid PED were confluent drusen, with a

72 focal area of PED involving the macular area, with a minimum size of 1/2 disc diameter [25], and without CNV 
74 on the basis of fluorescein and indocyanine green angiography, which showed an exudative change with CNV. In the

75 current study, eyes with central geographic atrophy were excluded. We also recruited 20 eyes (20 subjects) as an

76 age-adjusted control group. The criteria for the eyes, including for the control eyes, were as follows: $\geq 1.0$ VA on a

77 Landolt chart, $<10$ small drusen $(<63 \mu \mathrm{m})$ within $3000 \mu \mathrm{m}$ of the center of the macula on the fundus photograph,

78 normal morphology of the fovea as seen with optical coherence tomography (OCT), and absence of central

79 geographic atrophy or CNV.

81 Medicine and adhered to the tenets of the Declaration of Helsinki. Written informed consent for research

82 participation was obtained from each subject before examination.

83 Each subject underwent a comprehensive ophthalmologic examination, including measurement of

84 best-corrected VA on a Landolt chart, determination of intraocular pressure, indirect ophthalmoscopy, and slit-lamp

85 biomicroscopy with a contact lens. In each subject, $45^{\circ}$ digital fundus photographs were obtained using a digital

86 fundus camera (TRC-50LX; Topcon, Tokyo, Japan; $3216 \times 2136$ pixels) after pupil dilatation and the macular area

87 was examined with a Spectralis HRA+OCT device (Heidelberg Engineering, Heidelberg, Germany). Each patient

88 with large drusen, drusenoid PED, or neovascular AMD underwent fluorescein and indocyanine green angiography

89 with a confocal laser scanning system (HRA-2; Heidelberg Engineering). In each eye, macular function was

90 examined by fundus-monitored microperimetry and fmERG recording.

91 Retinal sensitivity within the macular area was examined with a fundus-monitored microperimeter (Micro 
92 Perimeter 1 [MP1]; Nidek, Gamagori, Japan). A 4-2-staircase strategy with Goldmann III-sized stimuli was used, and

9357 stimulus locations within a $10^{\circ}$ radius were examined by microperimetry. Each stimulus was located according to

94 the measurement points on the Humphrey 10-2, with some additional points. The white background illumination was

95 set at $1.27 \mathrm{~cd} / \mathrm{m}^{2}$. The differential luminance, defined as the difference between the stimulus luminance and

96 background luminance, was $127 \mathrm{~cd} / \mathrm{m}^{2}$ at $0-\mathrm{dB}$ stimulation, and the maximum stimulus attenuation was $20 \mathrm{~dB}$. The

97 stimulus duration was 200 milliseconds (ms), and the fixation target varied in size according to the VA of the patient.

98 There were 17 and 37 measurement points within the central circles with radii of $4^{\circ}$ and $8^{\circ}$, respectively.

The fmERG recording procedure has been previously described in detail $[8,9]$. Briefly, after maximal

dilatation of the pupils of both eyes, a Burian-Allen bipolar contact lens electrode (Hansen Ophthalmic Laboratories,

Iowa City, IA, USA) was placed in the conjunctival sac of each eye under topical anesthesia. A chloride silver

electrode was attached to the left earlobe to serve as the ground electrode. The fmERG was elicited by_circular

stimuli positioned on the macular area, using a prototype of the ER-80 (Kowa, Tokyo, Japan), which consisted of an

104 infrared camera (Kowa) and a stimulation system (Mayo Corporation, Nagoya, Japan). The luminance values of the

white stimulus light and the background illumination were 181.5 and $6.9 \mathrm{~cd} / \mathrm{m}^{2}$, respectively. The stimulus within the

$7.5^{\circ}$-radius circle was centered on the fovea, as observed through the infrared camera. The fmERG was recorded 
110 digitized at $10 \mathrm{kHz}$ with a band-pass filter of 5-500 $\mathrm{Hz}$ for the a-wave and the b-wave. The amplitudes of the a- and

111 b-waves were measured from baseline to the peak of the a-wave and from the trough of the a-wave to the peak of the

112 b-wave, respectively. Latency was defined as the time from the beginning of stimulation to the peak of each

113 component.

114 For the OCT images, the foveal thickness in each eye was determined in the following 2 ways: the distance

115 between the internal limiting membrane (ILM) and the outer border of the RPE or the distance between the ILM and

116 the Bruch membrane. In eyes with drusenoid PED, we also measured the height and area of the PED. For the

117 sequential OCT images, the height of the PED was defined as the maximal distance between the outer border of the

118 RPE and the Bruch membrane (sometimes outside the fovea). For the late-phase indocyanine green angiogram, the

119 area of the PED was measured using software built into the HRA-2. Briefly, drusenoid PED was observed as a dark

120 area on the late-phase indocyanine green angiogram, and the edge of this central dark area was traced manually. The

121 surrounding small dark lesions (drusen) isolated from the central PED were not included. Statistical analysis was performed using PASW Statistics version 17.0 software (SPSS, Chicago, IL, USA). All values were expressed as means \pm standard deviations. The best-corrected VA was measured using a Landolt chart

124 and converted to the logarithm of the minimum angle of resolution (logMAR). To clarify differences from the

125 healthy controls, all mean values between groups were compared using 1-way analysis of variance and post hoc

126 Dunnet tests. Bivariate analysis was done with the Pearson product moment correlation. 


\section{Results}

129 Table 1 shows the characteristics of the study populations. Although the controls ( $82.0 \pm 3.2$ years) were significantly

130 older than the patients with neovascular AMD (77.3 \pm 6.9 years, $P=0.019)$, there was no significant difference in the

131 gender or lens status of groups. In the control group, 13 eyes had small drusen in the macular area and 7 had no

132 drusen. All eyes showed good macular function (Fig. 1).

All functional parameters were measured, with VA, fmERG, and microperimetry showing significant variation

134 between the groups (Table 1). All eyes with neovascular AMD showed marked morphologic changes in the

135 neurosensory retina. In this group, cystoid macular edema was seen in 4 eyes (21\%), serous retinal detachment, in 14

136 eyes (74\%), and PED, in 17 eyes (89\%); foveal thickness of the neurosensory retina (384 $\pm 256 \mu \mathrm{m})$ was

significantly increased compared with the control eyes $(224 \pm 27 \mu \mathrm{m})$ (Fig. 2). Consistent with these morphologic

changes, macular function (VA, fmERG, and microperimetry) was significantly deteriorated in the neovascular AMD

group (Figs. 3 and 4).

$14440 \mu \mathrm{m}$ ) was no different from that in the control group (Fig. 2). In this large drusen group, while retinal sensitivity at 
microperimetry) were preserved (Figs. 3 and 5).

In the drusenoid PED group, all eyes had drusenoid PED of at least 1/2 disc diameter within the macular area.

148 The mean area of the PED was $4.78 \pm 3.74 \mathrm{~mm}^{2}$ and the mean height was $266 \pm 178 \mu \mathrm{m}$. In eyes with drusenoid

149 PED, the foveal thickness between the ILM and the Bruch membrane (377 $\pm 164 \mu \mathrm{m})$ was significantly greater than

150 that in the control eyes (224 $\pm 27 \mu \mathrm{m}, P=0.013)$. However, the structure of the neurosensory retina was well

151 preserved, and the foveal thickness between the ILM and RPE (200 $\pm 49 \mu \mathrm{m})$ did not differ from that in the control

152 group (Fig. 2). On the other hand, the macular function of these eyes was significantly deteriorated. VA, amplitude of

153 the a-wave and of the b-wave, and retinal sensitivity measured with the MP1 were significantly decreased when

154 compared with the control eyes (Figs. 3 and 6). Table 2 shows the correlation between the size of the drusenoid PED

155 and macular function and between the area of the PED and the latency of the a-wave and the b-wave, and retinal

156 sensitivity within the central $4^{\circ}$ or $8^{\circ}$. The height of the PED was negatively correlated with retinal sensitivity within

157 the central $4^{\circ}$ and $8^{\circ}$ areas (Fig. 7).

\section{Discussion}

160 Eyes with neovascular AMD often have a severe decrease in VA. In addition, because such eyes often show serous

161 retinal detachment, subretinal hemorrhage, retinal edema, or PED in the macular area, they may well have a

162 reduction in function in the macular area. With the use of fmERG, Nishihara et al reported that, in eyes with

163 neovascular AMD, the amplitude of each wave was reduced to $29 \%$ to $35 \%$ of that of the control eyes [26]. With the 
164 use of microperimetry, Sulzbacher et al [24] and Hautamäki et al [27] reported more recently that retinal sensitivity

165 was markedly decreased within the area of CNV, macular edema, hemorrhage, subretinal fluid, or PED in eyes with

166 neovascular AMD. In our patients with neovascular AMD, cystoid macular edema was seen in 21\%, serous retinal

167 detachment was seen in 74\%, and PED was seen in 89\% of the patients, and thickness of the fovea in the

168 neurosensory retina was significantly increased. In eyes with neovascular AMD, severe macular dysfunction is based

169 on the morphologic changes caused by the exudative change resulting from the CNV.

171 cause a functional disturbance in the macular area. So far, several electrophysiologic assessments have been

172 performed to study the macular function in eyes with drusen [23, 28-33]. Falsini et al documented an abnormality of

173 the focal ERG threshold in eyes with more than 20 soft drusen [33], although they did not investigate the correlation

174 between each drusen and the local sensitivity loss. With the use of microperimetry, Midena et al reported that retinal

175 sensitivity in eyes with large drusen $(>125 \mu \mathrm{m})$ was severely deteriorated[16]. Iwama et al reported that eyes with

176 confluent soft drusen often show focal areas with reduced retinal function consistent with irregularity of the RPE line

177 or of the junction between the inner and outer segments of the photoreceptors [34]. In the current study, while retinal

178 sensitivity at the central point was significantly decreased in eyes with large drusen, the other parameters of macular

179 function (VA, fmERG, and microperimetry) were well preserved. Although we did not assess function at each point,

180 retinal function may be focally deteriorated, consistent with the drusen. In addition, the area in which drusen are seen

181 may be involved in the reduction of macular function. 
183 drusen, often located in the center of the macula [35]. VA in eyes with drusenoid PED is reported to be relatively

184 good. In fact, in a recent report from the Age-Related Eye Disease Study, baseline VA in eyes with drusenoid PED

185 was $\sim 20 / 32$, with $~ 90 \%$ of eyes having VA better than 20/40 [35]. So far, however, little information is available on

186 the macular dysfunction caused by drusenoid PED. In the current study, VA, amplitude of the a-wave and b-wave,

187 and retinal sensitivity measured with the MP1 were significantly decreased when compared with the control eyes. In

188 addition, the area and height of the PED were correlated with the fmERG and with the retinal sensitivity within the

189 macular area—correlations that are consistent with the previously mentioned report of confluent drusen [34].

190 Photoreceptor damages, which could be observed as discontinuity of the junction of the inner and outer segments and

191 as presence of hyperreflective foci in the OCT image (Fig. 6) [36, 37], might result in decreased macular function in

192 eyes with drusenoid PED. Falsini et al also discussed that focal ERG sensitivity loss in eyes with drusen might result

193 from photoreceptor drop out [33], as could be slightly seen in the OCT images of eyes with large drusen in our study

194 (Fig. 5).

195 The prognosis of drusenoid PED was initially thought to be relatively good [38, 39]; however, a recent cohort

196 study reported a high rate of progression to more advanced AMD [35]. Roquet et al documented that presence of

197 metamorphopsia and drusenoid PED of greater than 2 disc diameters were risk factors of CNV occurrence within 2

198 years [25]. Recently, other research groups have reported results of pilot studies on the early treatment of drusenoid

199 PED without CNV by photodynamic therapy or by antivascular endothelial growth factor therapy [40-43]. 
200 Gallego-Pinazo et al successfully treated 6 patients with drusenoid PED using intravitreal ranibizumab.[41]

201 However, Krishnan and Lochhead reported rapid development of geographic atrophy after intravitreal injection of

202 pegaptanib in an eye with drusenoid PED [42]. In a recent report from the Age-Related Eye Disease Study, 19\% of

203 eyes with drusenoid PED developed central geographic atrophy and 23\% of these developed neovascular AMD [35].

204 When geographic atrophy develops in the extrafoveal region, VA measurement does not reflect a visual disturbance.

205 The effect of treatment for drusenoid PED remains controversial. Multimodal measurements of macular function

206 would be most helpful to evaluate the treatment efficacy of drusenoid PED.

There are various limitations to the current study. First, the eligible patients and controls in this study were all

208 Japanese, and the genetic background may well have influenced the characteristics of AMD, so our results should be

209 confirmed in another population. Second, the sample size of each group was small, so it is possible that we did not

210 detect small differences between groups. Third, the current study excluded central geographic atrophy, primarily

211 because this is a relatively rare feature of AMD in Japanese patients. Finally, this was a cross-sectional study, so we

212 could not offer any information regarding changes in macular function over time. Further longitudinal studies are

213 necessary to fully elucidate the macular function in eyes with AMD of various stages and to study the treatment

214 effects and the natural course of eyes with AMD, especially those with AMD in the early stage. Multimodal

215 evaluations of the entire macular function should be of great help in these endeavors. 
217 ACKNOWLEDGMENTS

218 None

219

220 
222 1. Friedman DS, O'Colmain BJ, Munoz B, Tomany SC, McCarty C, de Jong PT, et al. Prevalence of age-related macular degeneration in the United States. Arch Ophthalmol. 2004;122:564-72.

224 2. Bressler NM. Age-related macular degeneration is the leading cause of blindness. JAMA. 2004;291:1900-1.

$225 \quad$ 3. Resnikoff S, Pascolini D, Etya'ale D, Kocur I, Pararajasegaram R, Pokharel GP, et al. Global data on visual impairment in the year 2002. Bull World Health Organ. 2004;82:844-51.

4. Ishibashi $\mathrm{T}$ and on behalf of the L-JSG. Maintenance therapy with pegaptanib sodium for neovascular age-related macular degeneration: an exploratory study in Japanese patients (LEVEL-J study). Jpn J

Ophthalmol. 2013;57:417-23. photodynamic therapy combined with intravitreal ranibizumab for typical age-related macular degeneration and polypoidal choroidal vasculopathy. Jpn J Ophthalmol. 2013;57:283-93.

233 6. Yoshizawa C, Saito W, Hirose S, Kitamei H, Noda K, Ishida S. Photodynamic therapy combined with intravitreal bevacizumab and sub-tenon triamcinolone acetonide injections for age-related macular degeneration. Jpn J Ophthalmol. 2013;57:68-73. 
239 8. Ogino K, Tsujikawa A, Murakami T, Muraoka Y, Akagi-Kurashige Y, Ishihara K, et al. Evaluation of

macular function using focal macular electroretinography in eyes with macular edema associated with

branch retinal vein occlusion. Invest Ophthalmol Vis Sci. 2011;52:8047-55.

9. $\quad$ Ogino K, Tsujikawa A, Nakamura H, Miyamoto K, Murakami T, Muraoka Y, et al. Focal macular

2011;52:3514-20.

10. Miyake Y. Focal macular electroretinography. Nagoya J Med Sci. 1998;61:79-84.

11. Springer C, Bültmann S, Völcker HE, Rohrschneider K. Fundus perimetry with the Micro Perimeter 1 in

normal individuals: comparison with conventional threshold perimetry. Ophthalmology. 2005;112:848-54.

12. Rohrschneider K, Springer C, Bültmann S, Völcker HE. Microperimetry--comparison between the micro

perimeter 1 and scanning laser ophthalmoscope--fundus perimetry. Am J Ophthalmol. 2005;139:125-34.

13. Ritter M, Bolz M, Sacu S, Deak GG, Kiss C, Pruente C, et al. Effect of intravitreal ranibizumab in avascular

pigment epithelial detachment. Eye (Lond). 2010;24:962-8. 
257 16. Midena E, Vujosevic S, Convento E, Manfre A, Cavarzeran F, Pilotto E. Microperimetry and fundus autofluorescence in patients with early age-related macular degeneration. Br J Ophthalmol.

2007;91:1499-503.

17. Midena E, Radin PP, Pilotto E, Ghirlando A, Convento E, Varano M. Fixation pattern and macular sensitivity in eyes with subfoveal choroidal neovascularization secondary to age-related macular degeneration: a microperimetry study. Semin Ophthalmol. 2004;19:55-61.

18. Landa G, Su E, Garcia PM, Seiple WH, Rosen RB. Inner segment-outer segment junctional layer integrity and corresponding retinal sensitivity in dry and wet forms of age-related macular degeneration. Retina.

19. Dinc UA, Yenerel M, Gorgun E, Oncel M. Assessment of macular function by microperimetry in intermediate age-related macular degeneration. Eur J Ophthalmol. 2008;18:595-600. macular degeneration in patients with central field loss: different effects on maximum reading speed. Invest Ophthalmol Vis Sci. 2011;52:2417-24. of the loading regimen with intravitreal ranibizumab in neovascular age-related macular degeneration. $\mathrm{Br} \mathrm{J}$ Ophthalmol. 2010;94:185-9. 
with the micro perimeter 1 after photodynamic therapy for polypoidal choroidal vasculopathy. Am J

Ophthalmol. 2007;143:984-94.

23. Falsini B, Serrao S, Fadda A, Iarossi G, Porrello G, Cocco F, et al. Focal electroretinograms and fundus appearance in nonexudative age-related macular degeneration: quantitative relationship between retinal morphology and function. Graefes Arch Clin Exp Ophthalmol. 1999;237:193-200.

24. Sulzbacher F, Kiss C, Kaider A, Eisenkoelbl S, Munk M, Roberts P, et al. Correlation of SD-OCT features and retinal sensitivity in neovascular age-related macular degeneration. Invest Ophthalmol Vis Sci. detachment in age related macular degeneration. Br J Ophthalmol. 2004;88:638-42. electroretinograms in eyes with wet-type age-related macular degeneration. Invest Ophthalmol Vis Sci. exudative age-related macular degeneration lesion and focal retinal sensitivity. Acta Ophthalmol. doi:10.1111/j.1755-3768.2012.02556.x. 
morphology and perimetric sensitivity. Ophthalmic Res. 2009;41:194-202.

29. Li J, Tso MO, Lam TT. Reduced amplitude and delayed latency in foveal response of multifocal

electroretinogram in early age related macular degeneration. Br J Ophthalmol. 2001; 85:287-90.

30. Gerth C, Delahunt PB, Alam S, Morse LS, Werner JS. Cone-mediated multifocal electroretinogram in

age-related macular degeneration: progression over a long-term follow-up. Arch Ophthalmol.

2006;124:345-52.

31. Feigl B, Brown B, Lovie-Kitchin J, Swann P. Functional loss in early age-related maculopathy: the

ischaemia postreceptoral hypothesis. Eye (Lond). 2007;21:689-96.

32. Feigl B, Brown B, Lovie-Kitchin J, Swann P. Cone- and rod-mediated multifocal electroretinogram in early

33. Falsini B, Fadda A, Iarossi G, Piccardi M, Canu D, Minnella A, et al. Retinal sensitivity to flicker

modulation: reduced by early age-related maculopathy. Invest Ophthalmol Vis Sci. 2000;41:1498-506. 
311 36. Ogino K, Murakami T, Tsujikawa A, Miyamoto K, Sakamoto A, Ota M, et al. Characteristics of optical coherence tomographic hyperreflective foci in retinal vein occlusion. Retina. 2012;32:77-85.

313

37. Bolz M, Schmidt-Erfurth U, Deak G, Mylonas G, Kriechbaum K, Scholda C. Optical coherence tomographic hyperreflective foci: a morphologic sign of lipid extravasation in diabetic macular edema.

Ophthalmology. 2009;116:914-20.

38. Hartnett ME, Weiter JJ, Garsd A, Jalkh AE. Classification of retinal pigment epithelial detachments associated with drusen. Graefes Arch Clin Exp Ophthalmol. 1992;230:11-9.

39. Casswell AG, Kohen D, Bird AC. Retinal pigment epithelial detachments in the elderly: classification and outcome. Br J Ophthalmol. 1985;69:397-403.

40. Querques G, Bux AV, Delle Noci N. Foveal geographic atrophy following intravitreal pegaptanib sodium (Macugen) for drusenoid pigment epithelium detachment. Eur J Ophthalmol. 2009;19:890-3. ranibizumab for symptomatic drusenoid pigment epithelial detachment without choroidal neovascularization in age-related macular degeneration. Clin Ophthalmol. 2011;5:161-5.

42. Lee NY, Kim KS. Photodynamic therapy treatment for eyes with drusenoid pigment epithelium detachment.

Korean J Ophthalmol. 2008;22:194-6. 
Fig. 1 Macular function in a healthy control eye. Retinal sensitivity map obtained by microperimetry (a) and focal macular electroretinogram (b). White arrowhead = beginning of stimulus; yellow arrow = amplitude of each wave of

Fig. 2 Foveal thickness of control eyes, eyes with large drusen, eyes with drusenoid pigment epithelial detachment, and eyes with neovascular age-related macular degeneration. ${ }^{*} P<0.05, \dagger P<0.01, \ddagger P<0.0001$, compared with control eyes. $P$ values were calculated by the Dunnet test. ILM indicates internal limiting membrane; RPE, retinal

Fig. 3 Macular function measured with multimodality in control eyes, eyes with large drusen, eyes with drusenoid

341 pigment epithelial detachment, and eyes with neovascular age-related macular degeneration. ${ }^{*} P<0.05, \dagger P<0.01$,

$342 \ddagger P<0.0001$, as compared with control eyes. $P$ values were calculated by the Dunnet test. LogMAR indicates

343 logarithm of the minimum angle of resolution; PED, pigment epithelium detachment; AMD, age-related macular

344 degeneration

346 Fig. 4 Macular function in an eye with neovascular age-related macular degeneration. (a). Fundus photograph 
347 shows submacular hemorrhage (0.15 on a Landolt chart, OD). (b, c) Fluorescein and indocyanine green angiograms

348 reveal subfoveal choroidal neovascularization. Horizontal (d) and vertical (e) sections obtained with OCT show

349 subretinal fluid. (f) Retinal sensitivity map obtained with microperimetry shows a substantial reduction of retinal

350 sensitivity in the macular function. (g) Focal macular electroretinogram shows a substantial reduction in amplitude of

351 all waves. Arrowhead = beginning of stimulus

353 Fig. 5 Macular function in an eye with large drusen. (a) Fundus photograph shows multiple large drusen in the

354 macular area (1.0 on a Landolt chart, OD). (b, c) Fluorescein and indocyanine green angiograms reveal no choroidal

355 neovascularization. Horizontal (d) and vertical (e) sections obtained with OCT show multiple large drusen beneath

356 and affecting the fovea. The junction of the inner and outer segments of photoreceptors (between the arrows) was

357 discontinued. (f) Microperimetry shows preserved retinal sensitivity within the macular area except for the fovea. (g)

358 Focal macular electroretinogram shows that the amplitude of all of the waves was relatively preserved. Arrowhead $=$

359 beginning of stimulus

360

361 Fig. 6 Macular function in an eye with drusenoid pigment epithelial detachment (PED). (a) Fundus photograph of

362 drusenoid PED under the fovea (0.7 on a Landolt chart, OD). (b) Fluorescein angiogram reveals no choroidal

363 neovascularization. (c) From the late-phase indocyanine green angiogram, the area of drusenoid PED was calculated

364 as $6.26 \mathrm{~mm}^{2}$. Horizontal (d) and vertical (e) sections obtained with OCT show drusenoid PED. The height of the 
365 PED was $258 \mu \mathrm{m}$. The red arrow indicates hyperreflective foci. (f) Retinal sensitivity map obtained with

366 microperimetry shows a marked reduction in retinal sensitivity consistent with drusenoid PED. (g) In the focal

367 macular electroretinogram, the amplitude of each wave was reduced to $60 \%$ - $75 \%$ of normal amplitudes. Arrowhead

368 = beginning of stimulus

370 Fig. 7 Scattergram of the size of the drusenoid pigment epithelial detachment and macular functions measured with

371 focal macular electroretinogram or microperimetry. PED indicates pigment epithelium detachment 
373

TABLE 1. Background, foveal thickness, and macular function of control eyes, eyes with large drusen, eyes with drusenoid pigment epithelial detachment, and eyes with neovascular age-related macular degeneration.

\begin{tabular}{|c|c|c|c|c|c|}
\hline & Controls & Large Drusen & Drusenoid PED & Neovascular AMD & $P$ value \\
\hline Sex (male/female) & $16 / 4$ & $11 / 6$ & $18 / 0$ & $16 / 3$ & 0.054 \\
\hline Phakia/pseudophakia & $14 / 6$ & $9 / 8$ & $13 / 5$ & $12 / 7$ & 0.627 \\
\hline Age, y & $82.0 \pm 3.2$ & $80.7 \pm 5.2$ & $78.9 \pm 5.0$ & $77.3 \pm 6.9$ & 0.040 \\
\hline Visual acuity, $\log \mathrm{MAR}$ & $-0.07 \pm 0.07$ & $0.05 \pm 0.14$ & $0.16 \pm 0.18$ & $0.42 \pm 0.42$ & $<0.0001$ \\
\hline \multicolumn{6}{|l|}{ Foveal thickness, $\mu \mathrm{m}$} \\
\hline ILM to RPE & $224 \pm 27$ & $196 \pm 40$ & $200 \pm 49$ & $384 \pm 256$ & $<0.0001$ \\
\hline ILM to Bruch membrane & $224 \pm 27$ & $231 \pm 36$ & $377 \pm 164$ & $533 \pm 263$ & $<0.0001$ \\
\hline \multicolumn{6}{|l|}{ Amplitude of fmERG, $\mu \mathrm{V}$} \\
\hline a-wave & $1.73 \pm 0.65$ & $1.35 \pm 0.49$ & $1.21 \pm 0.67$ & $0.87 \pm 0.58$ & 0.0005 \\
\hline b-wave & $3.14 \pm 0.89$ & $2.55 \pm 0.91$ & $2.20 \pm 1.09$ & $1.37 \pm 1.04$ & $<0.0001$ \\
\hline \multicolumn{6}{|l|}{ Latency of fmERG, ms } \\
\hline a-wave & $23.18 \pm 1.28$ & $23.67 \pm 1.58$ & $24.39 \pm 1.77$ & $25.76 \pm 3.39$ & 0.040 \\
\hline b-wave & $42.05 \pm 2.27$ & $45.44 \pm 3.87$ & $45.22 \pm 3.71$ & $48.87 \pm 7.38$ & 0.0005 \\
\hline \multicolumn{6}{|l|}{ Retinal sensitivity, dB } \\
\hline center point & $14.78 \pm 3.52$ & $9.94 \pm 3.86$ & $3.82 \pm 3.43$ & $5.37 \pm 6.31$ & $<0.0001$ \\
\hline within $4^{\circ}$ & $16.50 \pm 2.01$ & $13.35 \pm 3.57$ & $6.83 \pm 4.39$ & $5.78 \pm 6.27$ & $<0.0001$ \\
\hline within $8^{\circ}$ & $16.13 \pm 2.10$ & $13.66 \pm 3.32$ & $9.19 \pm 3.94$ & $6.76 \pm 6.23$ & $<0.0001$ \\
\hline
\end{tabular}

PED pigment epithelium detachment, AMD age-related macular degeneration, fmERG focal macular electroretinogram, ILM internal limiting membrane, $R P E$ retinal pigment epithelium 
375

TABLE 2. Correlation between size of drusenoid pigment epithelium detachment and macular function.

\begin{tabular}{|c|c|c|c|c|}
\hline & \multicolumn{2}{|c|}{ Area of Drusenoid PED } & \multicolumn{2}{|c|}{ Height of Drusenoid PED } \\
\hline & $r$ & $P$ value & $r$ & $P$ value \\
\hline Visual acuity in logMAR & 0.058 & 0.820 & 0.432 & 0.074 \\
\hline \multicolumn{5}{|l|}{ Amplitude of fmERG } \\
\hline a-wave & -0.427 & 0.077 & -0.118 & 0.642 \\
\hline b-wave & -0.445 & 0.067 & -0.312 & 0.207 \\
\hline \multicolumn{5}{|l|}{ Latency of fmERG } \\
\hline a-wave & 0.635 & 0.006 & -0.090 & 0.732 \\
\hline b-wave & 0.530 & 0.029 & 0.100 & 0.702 \\
\hline \multicolumn{5}{|l|}{ Retinal sensitivity } \\
\hline center point & -0.472 & 0.056 & -0.423 & 0.091 \\
\hline within $4^{\circ}$ & -0.682 & 0.003 & -0.625 & 0.007 \\
\hline within $8^{\circ}$ & -0.761 & 0.0004 & -0.533 & 0.028 \\
\hline
\end{tabular}

PED pigment epithelium detachment, logMAR logarithm of the minimum angle of resolution, $f m E R G$ focal macular

electroretinogram

376 


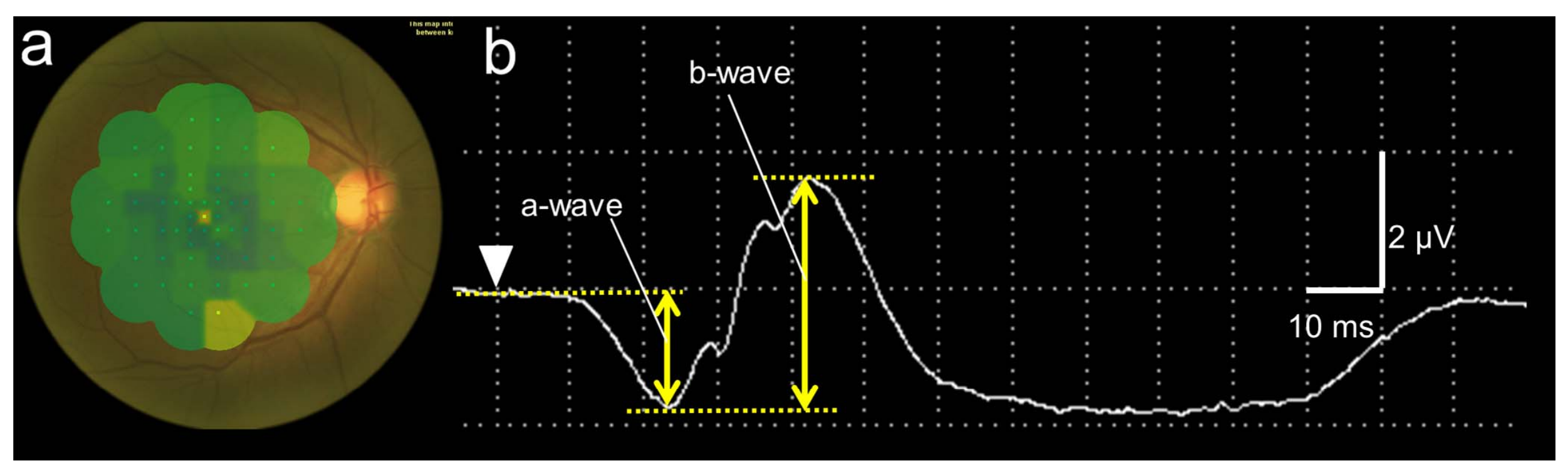

Fig. 1 

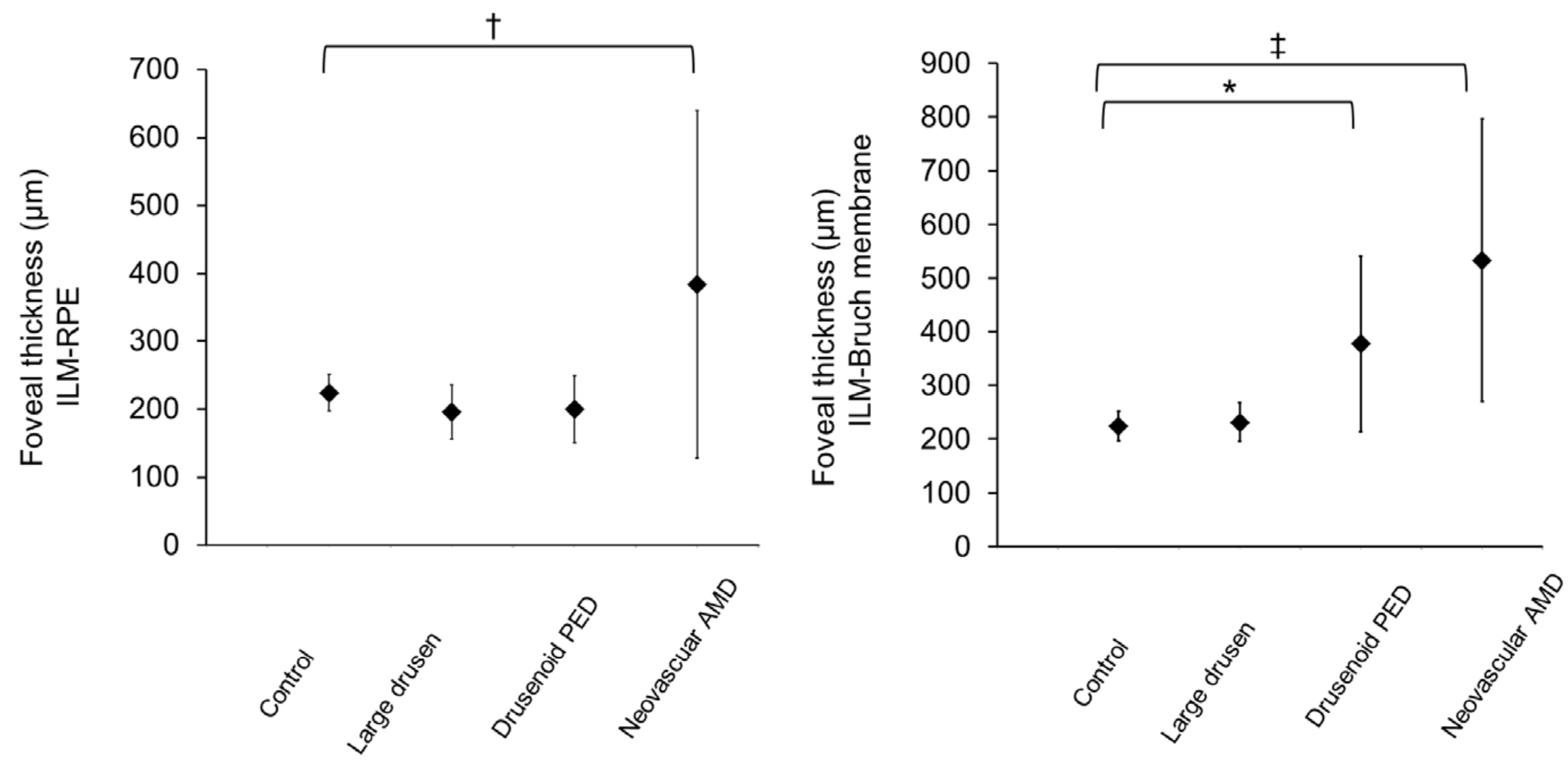

Fig. 2 

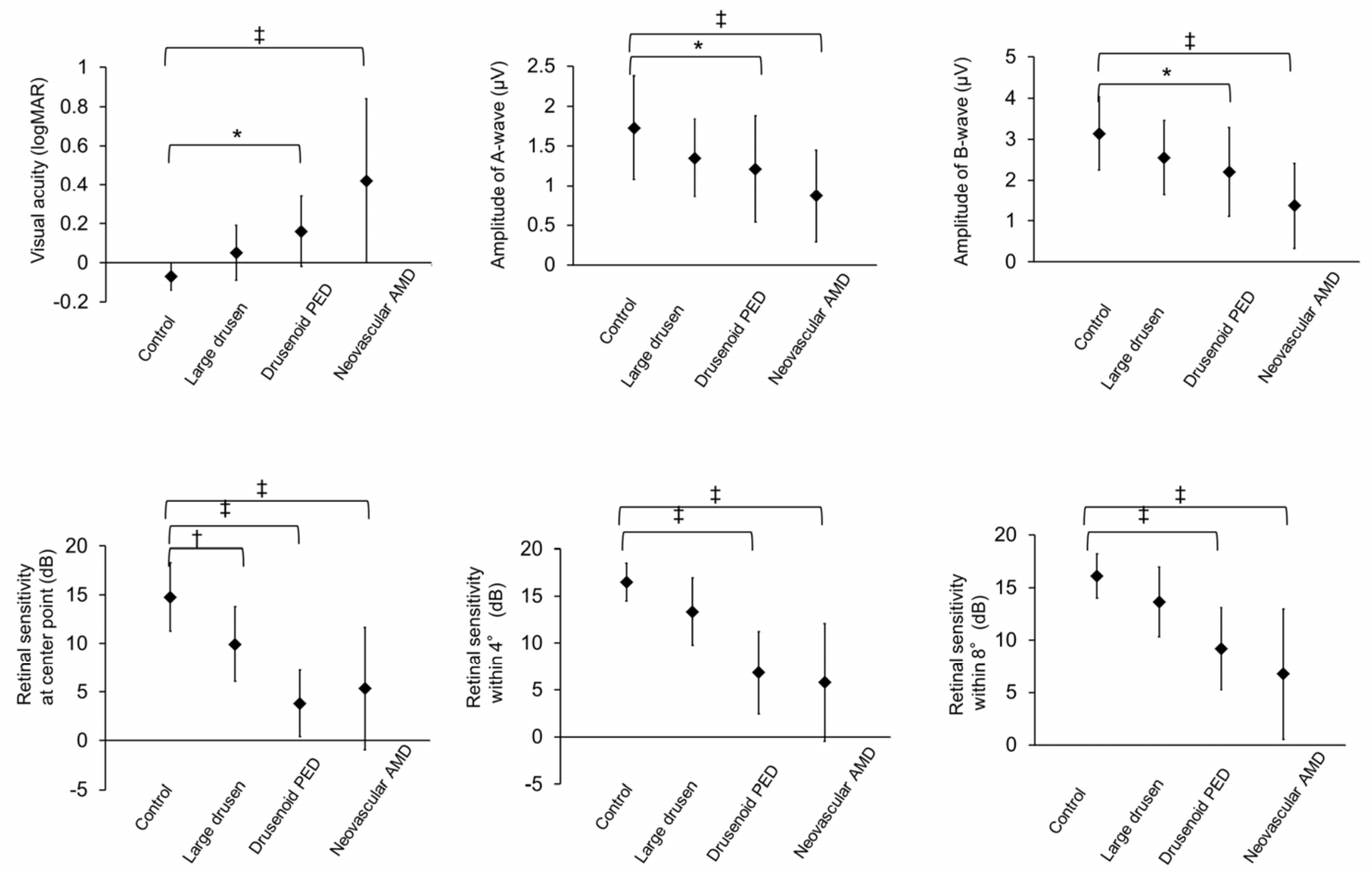

Fig. 3 

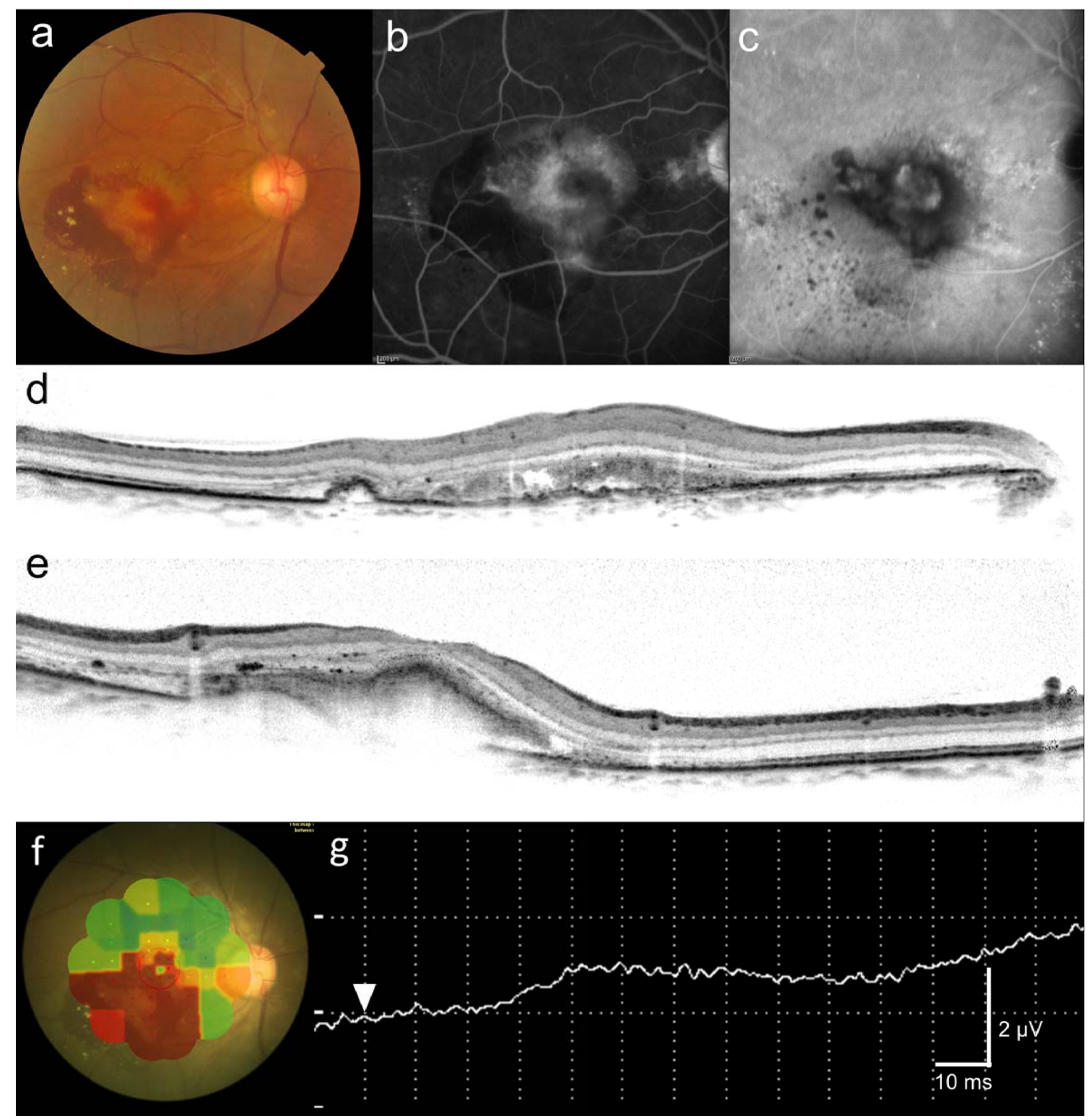

Fig. 4 

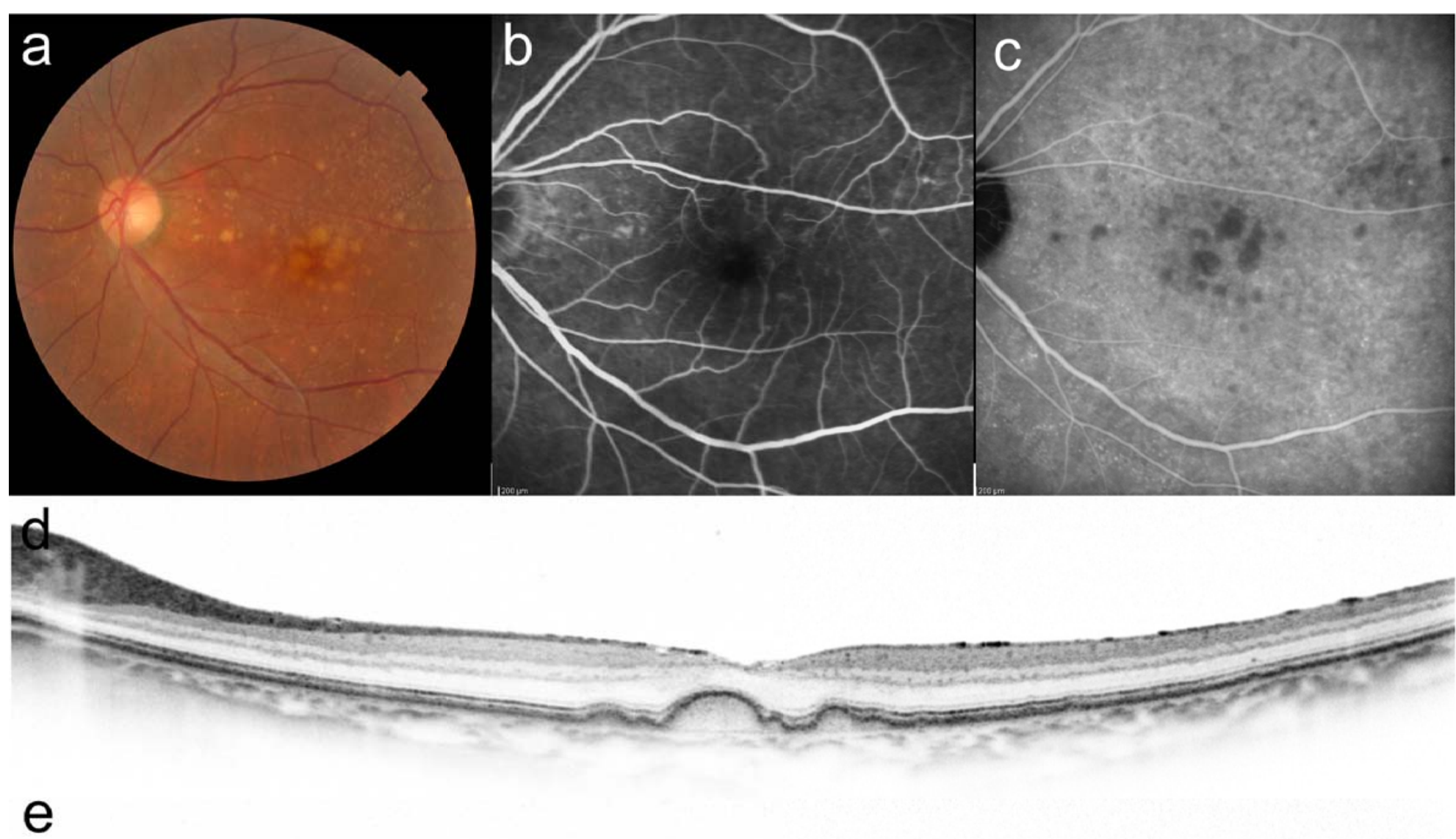

$=$

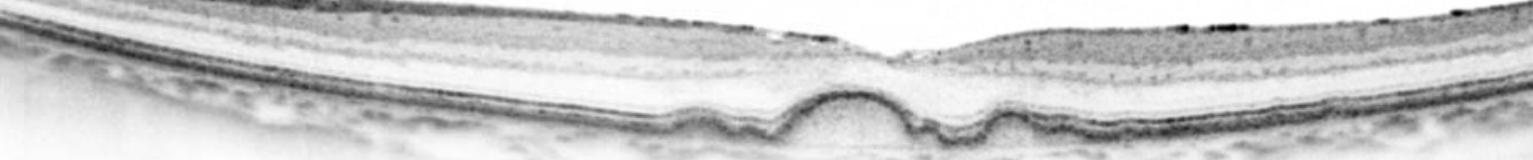

e

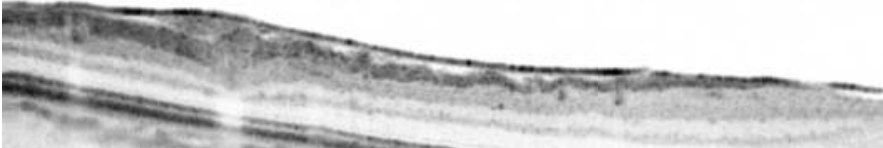

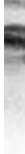

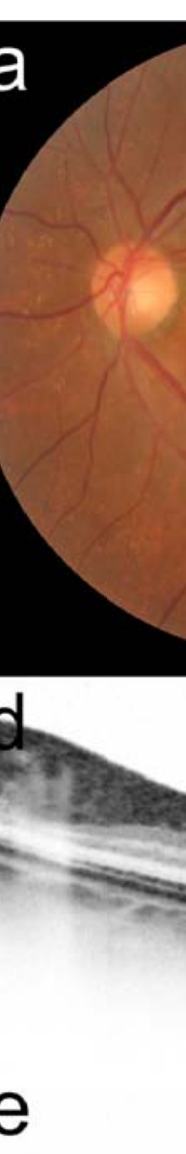



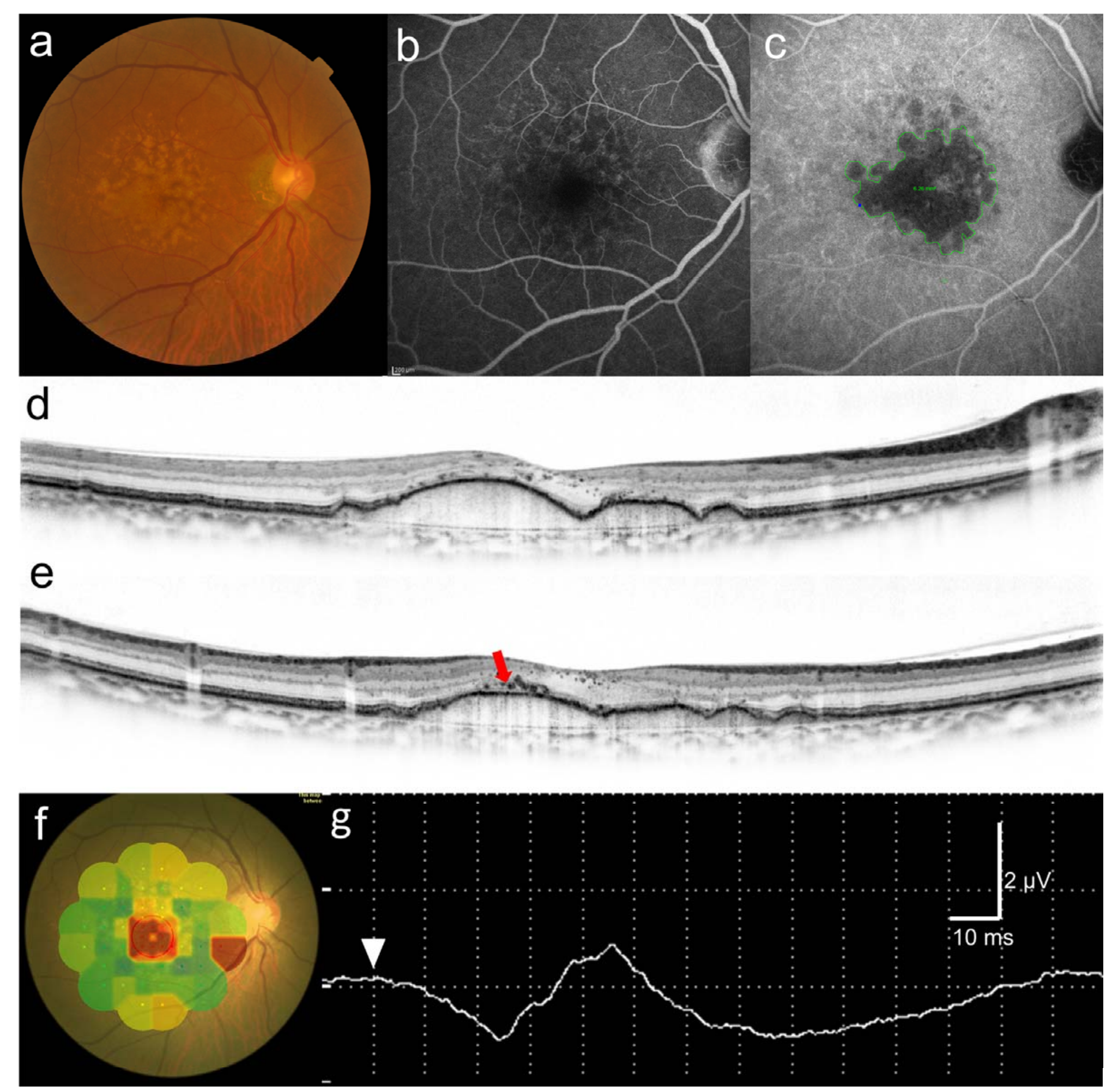

Fig. 6 